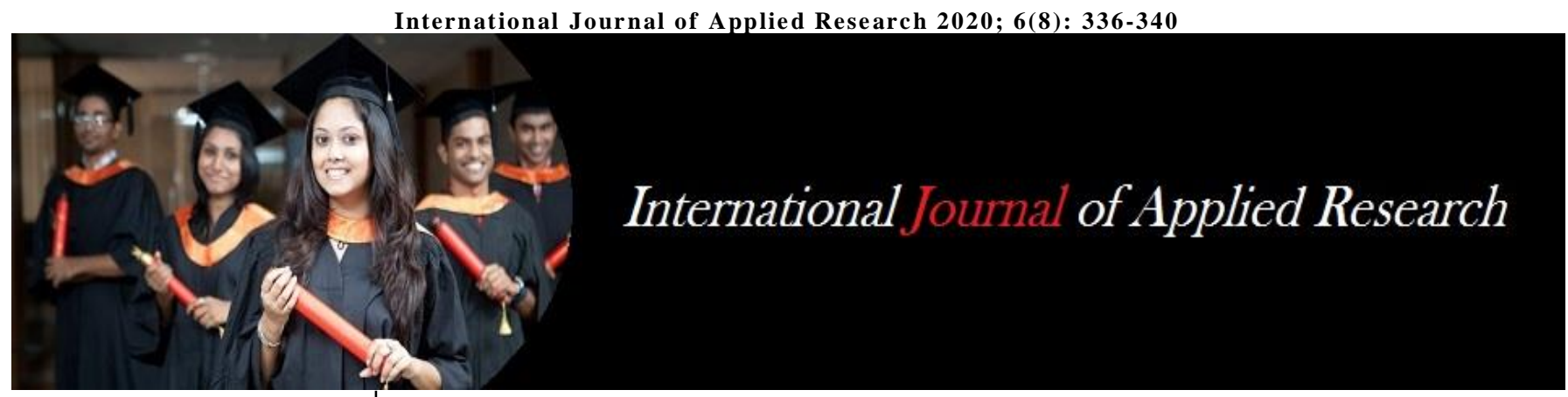

ISSN Print: 2394-7500

ISSN Online: 2394-5869

Impact Factor: 5.2

IJAR 2020; 6(8): 336-340

www.allresearchjournal.com

Received: 04-06-2020

Accepted: 06-07-2020

Sunita Argade

Msc. Nursing, Dr. DY Patil

College of Nursing, Pimpri,

Pune, Maharashtra, India

\section{Martha Raut}

Associate Professor,

Dr. DY Patil College of

Nursing, Pimpri, Pune,

Maharashtra, India

\section{Dr. Nisha Naik}

Associate Professor, Dr. DY

Patil College of Nursing,

Pimpri Pune, Maharashtra,

India

\section{Dr. Rupali Salvi}

Principal, Dr. DY Patil College

of Nursing, Pimpri Pune,

Maharashtra, India
Corresponding Author:

Sunita Argade

Msc. Nursing, Dr. DY Patil

College of Nursing, Pimpri,

Pune, Maharashtra, India

\section{A study to assess the effect of deep breathing exercise on maternal and fetal outcome among high risk mothers in selected hospital}

\author{
Sunita Argade, Martha Raut, Dr. Nisha Naik and Dr. Rupali Salvi
}

\section{Abstract}

Introduction: Giving birth should be your greatest achievement not your greatest fear. "In many languages the words for spirit and breath are one and the same Sanskrit prana, Hebrew ruach, Greek pneuma, Latin spiritus). Native American among others, believe that life enters the body with the first breath, not at the moment of birth or of conception. In this view the fetus and newborn have a kind of vegetative life, uninvited with spirit until the breath cycle begins. Breathing is the bridge between mind and body, the connection between consciousness and unconsciousness, the movement of spirit in matter. Breath is the key to health and wellness, a function we can learn to regulate and develop in order to improve our physical, mental and spiritual well-being.

Methods research approach

Research approach: Quantitative research approach. Research design used was experimental, purposive survey research design. The conceptual framework based on General System Model was used for the study which is designed by Von Bertalanffy (1968). The setting for this study was the selected in antenatal care unit, Dr. D.Y. Patil Hospital, \& Research Center, Pimpri, Pune. Nonprobability convenience sampling technique was used for 60 samples. The tool developed which includes

Section 1: The demographic variables

Section 2: It consists of six clinical profiles including details regarding the duration of the teaching program of deep breathing exercise.

Section 3: In observational checklist, there here total 5 vital aspects observed i.e. Mainly temperature, pulse, respiration blood pressure, and fetal heart sound. Tool validity was done and tool found reliable. Study found feasible after pilot study.

Results: The majority of the findings related to temperature observation between pre average and standard deviation $37.2(0.76)$, pulse 76 (4.09), respiration 17.3 (1.30), systolic blood pressure 130.15 (15.211), diastolic blood pressure 77.35 (3.065) \& fetal heart sound 136.75 (7.261) of the high risk anemia antenatal mothers were seen that it is highly significant. findings related to temperature observation between preaver age and standard deviation $37.2(0.7678)$ post $37.505(0.6605)$, pulse pre76(4.0911), post 78.65(2.4121s), respiration pre $17.3(1.30)$ post $18.35(1.755)$,systolic blood pressure pre130.5(15.212) post 126.85(10.489), diastolic blood pressure pre77.35(3.0655) post 76.9 (3.5229), fetal heart sound pre 136.75(7.2611) post 143.85(3.265) high risk anemic antenatal mothers were seen that it was highly significant. findings related to temperature observation between pre average and standard deviation 37.5 (0.512), pulse76.45(2.60), respiration16.8(1.23), systolic blood pressure 140 (8.583), diastolic blood pressure pre79.95 (3.51), fetal heart sound 133.9(6.896) high risk intra uterine growth retardation antenatal mothers were seen that it was highly significant. Since pvalue corresponding to education is $\mathrm{p}=0.001$ level of highly significant,(less than 0.05 ), education was found to have highly significant association with the deep breathing exercise by using high risk antenatal mothers.

Conclusion: The overall experience of conducting this study was new learning experience for the investigator it has been observed that temperature, pulse, respiration blood pressure fetal heart technique. Since $p$-value corresponding education is $(p=0.001)$ small (less than0.05) level of significant, education was found to have highly significant with the deep breathing exercise technique by using high risk antenatal mothers.

Keywords: Assess, effect, deep breathing exercise, maternal outcome, fetal outcome, high risk mother, anemia, intra-uterine growth retardation, hypertension

\section{Introduction}

Tuberculosis transmission occurs through droplet nuclei containing Mycobacterium tuberculosis, which are expelled by smear-positive pulmonary TB patients when coughing and sneezing, and remain suspended in the air Inhalation of such aerosols may lead to

$$
\sim 336 \sim
$$


infection After close contact with an infectious case, 30$50 \%$ of exposed susceptible contacts acquire latent TB infection. This can be determined by the tuberculin skin test or/and Interferon Gamma Release Assays After this first infection, active TB may occur immediately However, for the majority of cases, the initial infection remains clinically silent and microbiologically latent Approximately $10 \%$ of the infected individuals will progress. Robert Koch first identified Mycobacterium tuberculosis as the causative organism of tuberculosis in1882. It was however referred to as Koch's bacillus till Lehmann and Neumann gave the generic name Mycobacterium meaning fungus bacterium due to mould-like growth of the bacillus in liquid medium Myco bacterium tuberculosis is the etiologic agent of tuberculosis in humans and closely related Mycobacterium bovine

\section{Research design}

The research design selected for the study was an adopted experimental, purposive survey research design.

\section{Research setting}

The present study was conducted in the selected the study was conducted at the Dr. D.Y. Patil Hospital, \& Research Center, Pimpri.

\section{Population}

The population of the present study comprised of group high risk antenatal mothers which is included in deep breathing exercise in the selected antenatal care unit.

\section{Sample}

In the present study the samples were high risk antenatal mothers of D.Y. Patil Hospital\& Research Centre Pimpri, and Sahyadri Hospital, Pune.

\section{Sample size}

The Sample size consisted of 60 antenatal high risk mothers.

\section{Sample technique}

In the present study the sample was collected through Non probability convenience Sampling Technique.

\section{Criteria for selection of sample \\ Inclusion criteria}

1. Antenatal mothers who were anemia, intra uterine growth retardation \& hypertensive.

2. Antenatal mothers who were the age group 19-35 yrs.

3. Antenatal mothers who were available at the time of data collection.

4. Antenatal mothers who were able to read and write Marathi, Hindi and English

\section{Exclusion criteria}

1. Antenatal mothers were not willing to participate in the study.

2. Antenatal mothers who were on other alternative therapy like yoga.

\section{Development of tool}

Tool is a method, technique, device or a form design the tool to guide the observation to collect assess or record and measure the collected data in a systematic and uniform manner. Clinical teaching program of deep breathing exercise. Observational checklist for assessing the temperature, pulse, respiration, blood pressure and fetal heart sound. Review of literature. Personal consultation and discussion with the experts. Preparation of the tool. Content validity of the tool. The Investigator developed an observational checklist for assessing temperature, pulse, respiration, blood pressure and fetal heart sound. Observation scale for assessing the vitals by using beep breathing exercise teaching program among patients with high risk antenatal mothers.

Description of the tool: In this study the tool consisted of:Section A: Demographic Performa

Section B: It consists of six clinical profiles including details regarding the duration of the teaching program of deep breathing exercise

Section C: In observational checklist, there here total 5vital aspects observed i.e. Mainly temperature, pulse, respiration blood pressure, and fetal heart sound.

\section{Validity}

Content validity is concerned with the sampling adequacy of the content area being measured. Content validity is of special relevance to individuals designing a test to measure knowledge in a specific content area. The content validity of an instrument is based on judgment; experts in the content area may be called on to analyze the items. The tool for content validity was sent to 25 experts from different specialties i.e. Obstetrics \& Gynecology 12, Gynecologist 3 and Statistician. The validity done by 19 experts. They were requested to give their opinions on the appropriateness and relevance of the items in the tools. They were requested to give their opinions on the appropriateness and relevance of the items in the tool.Formal written permission was obtained from Medical officer, Superintendent of Dr. D.Y. Patil Hospital and Research centre, Pimpri Chinchwad Muncipal Corporation, Pune. They were requested to give their opinion on the appropriateness\& relevance of items in the tool.

\section{Reliability of the tools}

Reliability was assessed by using Inter-rater method the researcher assessed degree to which test scores. A reliability of 0.70 indicates $70 \%$ consistencies in the parameters are classified in Observational Checklist. Reliability for observational checklist was done using inter-rater method. The Reliability Coefficient Cohen's Kappa was found to be for deep breathing exercise 0.75 for hence the tool was reliable.

\section{Ethical consideration}

- Researcher had obtained approval from appropriate review boards to conduct the study.

- Researcher had taken formal permission from high risk antenatal mother to conduct study.

- Only the samples who had signed the consent form are included in the study.

- Confidentiality of the data is maintained strictly.

\section{Plan for data collection}

- Ethical committee clearance

- Permission from the Corporator and Medical Officer of selected hospital. 
- Consent from high risk antenatal from selected hospital.

- The investigator approached the high risk antenatal mothers of selected samples, informed them regarding

The objectives of the study and obtained their informed consent after assuring the confidentiality of the data.

data collection was done among selected sample by using teaching program and observational checklist was

Developed by using inter-rater method.

\section{Pilot study}

The pilot study was conducted from 10/11/2018 to 19/11/2018, on 10 samples from Sahyadri Hospital Pune, to assess the feasibility of the study and to decide the plan for data analysis. Investigator approached the subjects, informed them regarding the objectives of the study and obtained consent after assuring the subjects about the confidentiality of the data. The data was collected through the demographic performs, Observational Checklist and vital parameters.

\section{Data analysis and interpretation}

For the analysis of demographic variable would be analyzed in terms of frequency and percentage was be calculated. Mean, Median, Mode, Standard deviation, Percentage, Distribution, Frequencies for assess the knowledge. Fisher's Exact Test' would be applied to determine the significance of findings. The findings would be documented in tables, graphs and diagrams.

\section{Result \\ Section I-Frequency and percentage distribution of selective demographic variables \\ In antenatal group in age18-24years were 11(18.3\%) age 25- 30 years were $24(40 \%)$ age $31-35$ years were $19(31.6 \%)$ age $36-40$ years were $6(10 \%)$, education primary were $7(11.6 \%)$ higher secondary were $34(56.6 \%)$ degree were above. 13(21.6.\%) other 5 (8.3\%). Income Rs. 10000 were 4 (6.6\%). Rs.110000-20000 were27(45\%), Rs.21000-30000, were22 (36.6\%).Rs.310000-350000were 7(11.6\%).Obstetric history abortion were $14(23.3 \%)$ prime gravid 31 (52.6\%), multipara 12(20\%), number of children one were 14 $(23.3 \%)$ two were $29(48.3 \%)$, three were $11(18.3 \%)$ nil were $4(6.6 \%)$, bad habit nail biting were $15(25 \%)$, tobacco chewing were 26(43.3\%). knowledge deep breathing exercise yes were $25(41.6 \%) \&$ no were $34(56.6 \%)$.}

Section II-1. Analysis data related to the observation reported by pre deep breathing exercise technique of vital parameters on high risk antenatal anemic mothers and fetal heart sound of fetus

Deep breathing exercise was the effective.in temperature observation between pre average and standard deviation 37.2(0.76), pulse76(4.09), respiration 17.3(1.30), systolic blood pressure130.15(15.211), diastolic the blood pressure 77.35(3.065) and fetal heart sound136.75(7.261)of the high risk anemia antenatal mothers were seen that it was highly significant.

2. Analysis of data related to the observation reported by using deep breathing exercise technique on high risk anemic antenatal mother's vital and fetal heart sound: Deep breathing exercise was the effective.in temperature observation between pre average and standard deviation $37.2(0.7678)$ post $37.505(0.6605)$, puls pre 76 (4.0911) post
78.65 (2.4121), respiration pre 17.3 (1.30) post 18.35 (1.755), systolic blood pressure pre 130.15 (15.212) post 126.85 (10.489), diastolic blood pressure pre 77.35 (3.0655) post 76.9 (3.5229) \& fetal heart sound pre 136.75 (7.2611), post $143.85(3.265)$ high risk anemic antenatal mothers were seen that it is highly significant.

3. Analysis of data related to the Observation reported by using deep breathing exercise technique on high risk anemic antenatal mother's vital and fetal heart sound

Deep breathing exercise was the effective. In temperature observation between pre average and standard deviation 37.2(0.7678) post 37.505(0.6605), pulse pre 76 (4.0911) post $78.65(2.4121)$, respiration pre17.3(1.30) post 18.35 (1.755), systolic blood pressure pre130.15(15.212) post 126.85 (10.489), diastolic blood pressure pre77.35(3.0655) post 76.9 (3.5229) \& fetal heart sound pre 136.75(7.2611), post $143.85(3.265)$ high risk anemic antenatal mothers were seen that it is highly significant.

Section III: Analysis of data related to find the observation score by using deep breathing exercise technique on high risk anemic, intra uterine growth retardation\& hypertensive antenatal mother's and fetal heart sound. Deep breathing exercise was the effective. temperature pre and post observation between verageand standard deviationpre 36.853(5.0481) and post 36.881 (5.0622), pulse pre 74.96 (10.309) post 76.07 (10.393), respiration pre16.765(2.464), post 18.508(2.9391),systolic blood pressurepre 136.76 (19.719), post1 27.56 (18.318), diastolic blood pressure pre 77.77 (10.67), post 72.185 (10.035) fetal heart sound pre131.59(18.561),post 142.26 (19.56) of anemia, intra uterine growth retardation\& hypertensive high risk antenatal mothers were seen that it was highly significant.

\section{Conclusion}

The overall experience of conducting this study was new learning experience for the investigator it has been observed that temperature, pulse, respiration blood pressure fetal heart technique. Since p-value corresponding education is $(\mathrm{p}=0.001)$ small (less than 0.05) level of significant, education was found to have highly significant with the deep breathing exercise technique by using high risk antenatal mothers.

\section{Discussion}

This study was designing to the experimental one group research designing; a non-probability convenience sampling technique used. The setting of the study was at Dr. D.Y. Patil Hospital and Research Center, Pimpri, Pune, Maharashtra, India. Existing high risk antenatal mothers reported by using deep breathing exercise technique assessed with observational checklist scale. The size of the sample was $(n=60)$. Sample divided in to three part. Anemia $(n=20)$, intra uterine growth retardation $(n=20)$ \& hypertension $(n=20)$. Sample In selected antenatal care unit according to inclusive and exclusive criteria. In present study in deep breathing exercise technique is effective for the high risk antenatal mothers. Since p-value corresponding to education is $(\mathrm{p}=0.001)$ level of highly significant, (less than 0.05). 


\section{Limitations}

- Data collection period was limited.

- Long term follow up could not be carried out due to time constraints.

- This study was limited only 60 samples; hence the finding cannot be generalized.

- Small samples from two Centers were used so generalization of this finding is limited.

- The study is only limited for the high risk antenatal mother admitted in Dr. D.Y. Patil, Hospital, Pune.

\section{Recommendations}

1. The study can be undertaken in different settings and different target population.

2. A similar study can be done to assess the effectiveness of antenatal exercises on behavioral responses during first stage of labor and outcome of labor.

3. The comparative study can be done in rural and urban area.

4. A study can replicate on large sample so thereby findings can be generalized.

5. The study can be taken in different setting and different target population such as health workers, nursing student, doctors, industrial employs and teachers.

6. A study can done on association between various demographic variables which were significant on large samples.

\section{Acknowledgement}

Firstly, I thank God for His abiding grace, which has made this possible. This effort in my academic pursuit would not have been a reality without constant support, guidance and encouragement rendered by a number of people, whosehelp, I specially recognize through this study with grateful gratitude. I thank my parents for their support. It is because of them that I have been able to drive the strength to complete this study. I heartily thank Mrs. Rupali Salvi, Principal, Dr. D.Y Patil College of Nursing for her support and expert guidance, My research coordinator Dr. Mrs. Nisha Naik and guide Mrs. Martha Raut, Assoc. Professor, Dr. D.Y Patil College of Nursing, for the expert positive and supportive guidance. During my stressful moment, they were like rising sun casting brightness, hope and bringing life to the gloomiest Their humour, wisdom has been the positive aspect of any situation was the propelling force which culminated in this dissertation, the crowning glory of all the endeavors. I am thankful to them for their inspiration, constant guidance, sustained patience, support and moreover encouragement right from the inception until the completion of study. I am deeply indebted to Dr. Mrs. Nisha Naik, Research Co-coordinator for her expert guidance, sustained patience and valuable suggestion. I take this opportunity to express my sincere gratitude towards the entire faculty Dr. D. Patil College of Nursing, Pimpri, Pune-18 as well as the administrative staff for their support and assistance throughout the study period. I take this opportunity to thank all experts in the field of Obstetric and Gynecologist Nursing, and statistician for their valuable suggestions and validation of the data collection instrument and plan. My sincere thanks to the antenatal care unit of Dr. D.Y. Patil, Hospital and Research Centre that allowed and supported to conduct my pilot study in antenatal care department in Sahyadri Hospital, Pune. I am grateful to the antenatal care unit of Dr. D.Y Patil, Hospital Pimpri, Pune-18 who allowed and supported me to conduct this study. Would also like to convey my sincere thanks to Mrs. Vaishali Chirmude, Statistician for assisting me with analysis of data.

I would like to thank all participants who have made this study possible. Lastly, my sincere thanks to all colleagues, friends, family members, My Father Mr. Bhimrao More, My Mother Mrs. Laxmi More, My Husband Mr. Vijay Argade \& My Daughter Miss. Nupur Argadeand son Shantanu Argade and all well wishes for their good wishes for this study.

\section{References}

1. Preethi Jazna et al., Madurai. Asian J. Nur. Edu. and Research. 2016; 6(2):167-170.

2. Basvanthappa BT. Nursing Research $2^{\text {nd }}$ Edition Jaypee Publication, 2011

3. Chloe V, et al., Pregnancy and advanced maternal age. Progress in obstetrics and gynecology.17th ed. Philadelphia: Elsevier Publishers; 2006, 113-124

4. Wildschut HI. Pregnancy antecedents of high pregnancy. In: James DK, Steer PJ, Weiner CP, Gonik $\mathrm{B}$, editors. High risk pregnancy. Management options. 3rd ed. Philadelphia: Elsevier Publishers, 2006, 3-41.

5. Ling H, Sauve R, Nicholas B, Fergusson D, Martenal age, risk of stillbirth a systematic review. 2008; 178:165-172.

6. American College of Obstetrics and Gynecology Committee on Obstetric Practice. ACOG Committee opinion. Number 267, January 2002: exercise during pregnancy and the postpartum period. Obstet Gynecol 2002; 99:171-173

7. Hopkins SA, Baldi JC, Cutfield WS, McCowan L, Hofman PL. Exercise training in pregnancy reduces offspring size without changes in maternal insulin sensitivity. J Clin Endocrinol Metab 2010; 95:20802088

8. Herbert RD. Effective physiotherapy. Br Med J 2001; 323:788-790.

9. Ali A Gehan et al., Cairo, Egypt, Efficacy of antenatal exercises on maternal and neonatal outcomes in elderly primigravida 2015; 21(3):109-114

10. Ali A Gehan et al., Cairo, Egypt, Efficacy of antenatal exercises on maternal and neonatal outcomes in elderly primigravida $2015 ; 21(3): 109-114$.

11. Riva Rahl Benefits and risks of physical activity for pregnant women Article Online Conditions form 57, 2014

12. Preethi Jazna et al. Madurai. Asian J. Nur. Edu. and Research. 2016; 6(2):167- 170.

13. Hong Gong et al., effectiveness of yoga as an intervention in the management of prenatal depression Published online Feb 5. Doi US:10.1186/

14. Thamilmani R, Shylamma Job. Hypertension and Abdominal breathing exercise. 2009; 1(1):18-21.

15. Chang BS et al. Effects of abdominal breathing on anxiety, blood pressure. Korean J Women Health Nurse 2009; 15(1):32-42.

16. Elliott WJ et al. Graded blood pressure reduction in hypertensive outpatients Slows breathing. The Journal of Clinical Hypertension. October 2004; 6:553-556.

17. Gavish B. Device-guided breathing on hypertension. Israelfrom: 2013,http://www.ncbi.nlm.nih.gov/pubmed

18. Grossman E et al. Breathing- control lowers blood pressure. Journal of Human Hypertension. 2001; 15:263 
19. Ali A Gehan et al., Cairo, Egypt, Efficacy of antenatal exercises on maternal and neonatal outcomes in elderly primigravida 2015; 21(3):109-114.

20. Wanda Scherrer et al. Santo, Brazil, Effects of relaxation on depression level in women with high risk pregnancy-doi: 10.1590/15188345.1249.2806 2016; 24:2806.

21. Jiang Q et al. Effects of yoga intervention during pregnancy: Doi: 10.1055/s 0034-1396701.Publishers 333Seventh Avenue,2015 May NewYork,USA.22.Hilai Yuksel, Yasemin cayara. Effectiveness breathing exercises during the second Stage of labor on labor pain and duration: $2015 ; 15(6): 456-461$.

22. Majid Ravanbakhsh et al. Effect of Breathing Exercises on Breathing Pattern of pregnant women Ahvaz Judishapur, Iran, 2014.

23. Akshykumari Jahala, effectiveness of Lamaze breathing on laborpain and anxiety to wards labor outcome among primigravida mothers during labor road, Bhopal. March, 2017, 869.

DOI: $10.21088 /$ ijog.2321.1636.5117.2

24. Sevil Cicek et al., effects of breathing techniques training on duration of labor and anxiety levels of pregnant women, Kutahya, Turkey, 2017, 11.

25. Rakhshani et al. Systematic Review of yoga for pregnant women, current status and future directions. kathryn curtis, Aliza Weinrib, and Joel Katz, Toronto, Canada, 2012; http://dx.doi.org/10.1155/2012/715942.

26. Raziyeh Haddadi et, The Effect Breathing Exercises on Breathing Pattern of Pregnant women, 2014.

27. Eujim et al., Istanbul, Turkey Effect of Diaphragmatic Breathing Exercise on Psychological Parameters in Gestational Diabetes: A Randomised Controlled Trial. 2018 DOI:10.1016/j09.006.

28. Department of Nursing, et al., Cheonan, Korea. Effects of abdominal breathing on state anxiety, stress, and tocolytic dosage for pregnant women in Cheonan, Korea. 2010; 40(3):442-52.doi:10.4040/jkan.2010

29. Mahin Kamalifard et al. Efficacy of Massage Therapy and Breathing Techniques on Pain Intensity and Physiological Responses to Labor Pain; Published online 2012. doi: 10.5681/jcs.2012.011 Tabriz.

30. Isabel F et al. USA, Fetal response to abbreviated relaxation techniques, 2011.

31. Monk C et al. New work Effects of maternal breathing rate, Psychiatric status, and cortisol fetal heart rate, 2012.

32. Kathleen M. Gustafson et Kansas Fetal Cardiac Autonomic Control during Breathing and NonBreathing, 2012.

33. Polite, Beck Nursing Research and Principles and methods, $6^{\text {th }}$ Edition Philadelphi (us): Lippincott Company L, 2008. 